\title{
Comparison of SNR efficiencies and strain for cine DENSE using conventional EPI, flyback EPI and spiral k-space trajectories
}

\author{
Xiaodong Zhong ${ }^{1 *}$, Bruce S Spottiswoode ${ }^{2}$, Craig H Meyer ${ }^{3}$, Frederick H Epstein ${ }^{3}$ \\ From 2011 SCMR/Euro CMR Joint Scientific Sessions \\ Nice, France. 3-6 February 2011
}

\section{Introduction}

The original implementation of 2D cine DENSE (displacement encoding with stimulated echoes) employed a conventional EPI $k$-space trajectory for rapid data sampling. Follow-up studies used flyback EPI to reduce image artifacts. More recently a spiral $k$-space trajectory was utilized for improved SNR.

\section{Purpose}

To evaluate and compare SNR efficiencies and strain results of these three techniques for 2D cine DENSE imaging.

\section{Methods}

Cine DENSE pulse sequences were developed that employed different $k$-space trajectories, namely conventional bottom-up interleaved EPI, flyback bottom-up interleaved EPI, and interleaved spiral. They were compared in volunteers using a two-breath-hold protocol on a $1.5 \mathrm{~T}$ Siemens Avanto system with a four-channel chest coil. In accordance with protocols approved by the local institutional review board, 5 healthy volunteers were imaged. Identical parameters included pixel size $=$ $3.8 \times 3.8 \mathrm{~mm}^{2}$, slice thickness $=8 \mathrm{~mm}$, flip angle $=20^{\circ}$, cardiac phases $=15$, displacement encoding frequency $=$ 0.08 cycles $/ \mathrm{mm}$, two-point phase cycling, and throughplane dephasing frequency $=0.08$ cycles $/ \mathrm{mm}$ to suppress artifacts. Other parameters for conventional/ flyback EPI included image matrix $=72 \times 96$, TE $=$ $8.57 / 10.57 \mathrm{~ms}$, TR $=17.69 / 21.19 \mathrm{~ms}$, ETL $=8$, segments $=16$, sampling time per image $=99.8 \mathrm{~ms}$, heartbeats per breath-hold $=21 / 20$, and fat suppression by water

${ }^{1}$ Siemens Healthcare, Lilburn, GA, USA

Full list of author information is available at the end of the article excitation. For spiral, other parameters included image matrix $=96 \times 96, \mathrm{TE}=1.08 \mathrm{~ms}, \mathrm{TR}=17 \mathrm{~ms}$, interleaves $=6$, interleaves per heartbeat $=2$, sampling time per image $=66.8 \mathrm{~ms}$, heartbeats per breath-hold $=14$, and fat suppression by chemically-selective saturation pulses applied prior to displacement-encoding pulses. Based on the magnitude-reconstructed images, SNR efficiencies were calculated. Strain maps were also calculated.

\section{Results}

As shown in Figure 1, there is a small difference in SNR efficiencies between conventional and flyback EPI, which is due to their small TE and TR differences. In contrast, the SNR efficiency of spiral increases by about 33\% at early phases and about $79 \%$ at late phases compared to the EPI techniques, which is attributed to shorter TE and more efficient sampling. The circumferential strain

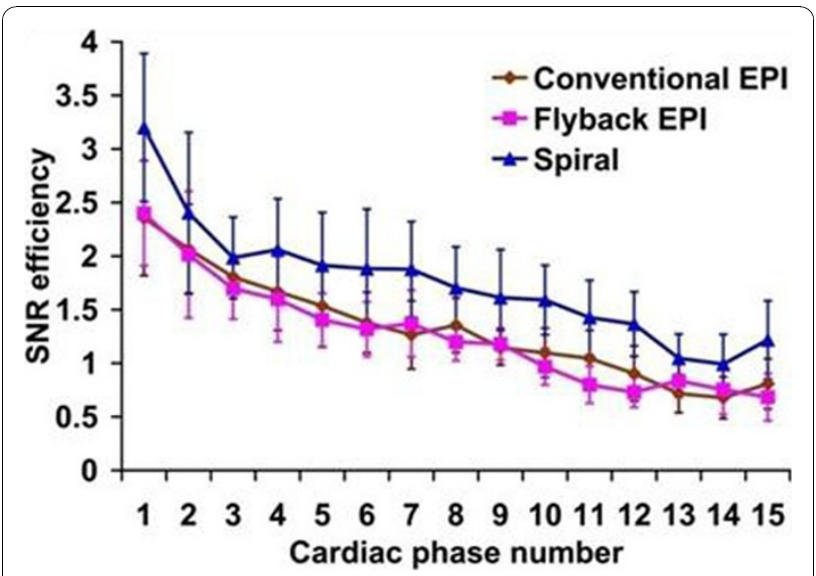

Figure 1 Mean SNR efficiency curves of three techniques.

\section{C) Biomed Central}

○ 2011 Zhong et al; licensee BioMed Central Ltd. This is an open access article distributed under the terms of the Creative Commons Attribution License (http://creativecommons.org/licenses/by/2.0), which permits unrestricted use, distribution, and reproduction in any medium, provided the original work is properly cited. 
Table 1 The end-systolic Ecc of mid-ventricular segments measured with three techniques

\begin{tabular}{lllllll}
\hline & Mid anterior & Mid lateral & Mid posterior & Mid inferior & Mid septum & Mid anterior septum \\
\hline Conventional EPI DENSE & $-0.20 \pm 0.02$ & $-0.22 \pm 0.04$ & $-0.19 \pm 0.05$ & $-0.18 \pm 0.02$ & $-0.17 \pm 0.01$ & $-0.17 \pm 0.02$ \\
Flyback EPI DENSE & $-0.19 \pm 0.03$ & $-0.22 \pm 0.05$ & $-0.21 \pm 0.03$ & $-0.18 \pm 0.02$ & $-0.14 \pm 0.01$ & $-0.15 \pm 0.02$ \\
Spiral DENSE & $-0.19 \pm 0.03$ & $-0.21 \pm 0.03$ & $-0.21 \pm 0.02$ & $-0.17 \pm 0.02$ & $-0.15 \pm 0.03$ & $-0.15 \pm 0.03$ \\
\hline
\end{tabular}

(Ecc) was calculated for mid-ventricular segments at end-systole (Table 1). One-way ANOVA analysis showed no statistically significant difference among three techniques.

\section{Conclusions}

Conventional EPI, flyback EPI and spiral cine DENSE produce similar strain results. Spiral cine DENSE provides improved SNR efficiency compared to the other two techniques.

\section{Author details}

${ }^{1}$ Siemens Healthcare, Lilburn, GA, USA. ${ }^{2}$ MRC/UCT Medical Imaging Research

Unit, University of Cape Town, Cape Town, South Africa. ${ }^{3}$ Biomedical

Engineering and Radiology, University of Virginia, Charlottesville, VA, USA.

Published: 2 February 2011

doi:10.1186/1532-429X-13-S1-P58

Cite this article as: Zhong et al:: Comparison of SNR efficiencies and strain for cine DENSE using conventional EPI, flyback EPI and spiral kspace trajectories. Journal of Cardiovascular Magnetic Resonance 201113 (Suppl 1):P58.

Submit your next manuscript to BioMed Central and take full advantage of:

- Convenient online submission

- Thorough peer review

- No space constraints or color figure charges

- Immediate publication on acceptance

- Inclusion in PubMed, CAS, Scopus and Google Scholar

- Research which is freely available for redistribution

Submit your manuscript at www.biomedcentral.com/submit 